\title{
MEGARA Optical design: the new integral field unit and multi-object spectrograph for the GTC 10m telescope
}

\author{
María-Luisa García-Vargas*a, Ernesto Sánchez-Blanco ${ }^{\mathrm{a}}$, Esperanza Carrasco ${ }^{\mathrm{b}}$, Armando Gil de Paz $^{\mathrm{c}}$, \\ Gonzalo Páez ${ }^{\mathrm{d}}$, Ana Pérez ${ }^{\mathrm{a}}$, Jesús Gallego ${ }^{\mathrm{c}}$, Francisco Sánchez ${ }^{\mathrm{e}}$ and José M. Vílchez ${ }^{\mathrm{f}}$ \\ ${ }^{a}$ FRACTAL SLNE, C/ Tulipán 2, p13-1A, E-28231 Las Rozas de Madrid (Spain); ${ }^{b}$ INAOE, C/ Luis \\ Enrique Erro 1, Tonantzintla, Puebla (México); 'Departamento de Astrofísica y CC. de la Atmósfera, \\ Fac. Ciencias Físicas, Universidad Complutense de Madrid, Avda. de la Complutense s/n, E-28040 \\ Madrid (Spain); ${ }^{d}$ CIO, Loma del Bosque 115, León, Gto., México CP 37154; ${ }^{\circ}$ DATSI, Facultad de \\ Informática, Universidad Politécnica de Madrid, Campus de Montegancedo, 28660 Boadilla del

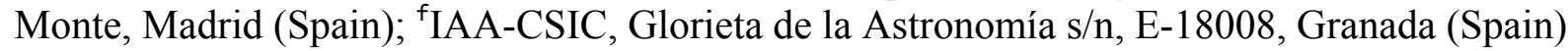

\begin{abstract}
We describe the optical design of MEGARA, the future optical Integral Field Unit (IFU) and Multi-Object Spectrograph (MOS) for the 10.4-m Gran Telescopio CANARIAS (GTC). MEGARA is being built by a Consortium of public research institutions led by the Universidad Complutense de Madrid (UCM, Spain) that also includes INAOE (Mexico), IAA-CSIC (Spain) and UPM (Spain).
\end{abstract}

Keywords: Instrumentation, spectrograph, optics, design, telescope, GTC, MEGARA

\section{INTRODUCTION}

MEGARA $^{1}$ (Multi-Espectrógrafo en GTC de Alta Resolución para Astronomía) is an optical Integral-Field Unit (IFU) and Multi-Object Spectrograph (MOS) designed for the GTC 10.4m telescope, in operation in La Palma (Spain). The MEGARA Consortium is lead by Universidad Complutense de Madrid (UCM, Madrid-Spain) and has as co-partners the Instituto Nacional de Astrofísica, Óptica y Electrónica (INAOE; Puebla, Mexico), the Instituto de Astrofísica de Andalucía (IAA-CSIC; Granada, Spain) and the Universidad Politécnica de Madrid (UPM; Madrid, Spain).

MEGARA has been already selected as the next optical instrument for GTC. The instrument passed its Preliminary Design Review in May 2012 and is progressing through a Critical Design Review in May 2013 for starting operations at the telescope at the end of 2015. A general view of MEGARA on the GTC telescope is shown in Figure 1. MEGARA has two main subsystems with optical components: the focal plane units at the Folded-Cass focal station, and the spectrograph, on the Nasmyth platform both connected through optical-fiber bundles. MEGARA offers two IFU modes with different bundles placed at the telescope focal plane: The Large Compact Bundle (LCB), covering $12 \operatorname{arcsec} \mathrm{x} 11$ arcsec with a spaxel size of 0.62 arcsec, making use of $100 \mu \mathrm{m}$-core optical fibers and the Small Compact Bundle (SCB) covering $9 \operatorname{arcsec} \times 7 \operatorname{arcsec}$ with a spaxel size of $0.42 \operatorname{arcsec}$ and $70 \mu \mathrm{m}$-core fibers. In addition, the MEGARA MOS mode will allow observing up to 100 objects in a region of $3.5 \operatorname{arcmin} \times 3.5$ arcmin around the two IFU bundles. Eight of these bundles will be devoted to the determination of the sky during the observation with the LCB IFU, so only 92 of these positioners will be available for MOS observations. Each of these bundles is composed by optical fibers and microlenses that allow converting the telescope focal ratio (f/17) into a faster beam (f/3) entering in the fibers to minimize the losses. Each fiber bundle ends in a pseudo-slit arrangement that feeds the spectrograph, acting as slit with the slit width set by the fiber core size. The spectrograph has a classical collimator-camera design with a pupil in which different dispersive elements (all of them based on Volume Phase Holographic -VPH- gratings) can be selected thanks to the use of a mechanism. A complete set of these 14 elements will be manufactured being 11 of them able to be simultaneously mounted. The design is different for each of them and we have called them LR, MR and HR gratings for Low, Medium and High resolution respectively. The different fiber core sizes of the bundles drive the different spectral

Optical Systems Design 2012, edited by Pablo Benítez, et al., Proc. of SPIE Vol. 8550, 85501C · @ 2012 SPIE

CCC code: $0277-786 / 12 / \$ 18 \cdot$ doi: $10.1117 / 12.2006270$ 
resolutions. Both the LCB IFU and MOS will provide spectral resolutions with $\mathrm{R}\left(\mathrm{EED}_{80}\right) \sim 5,500,10,000$ and 17,000 for LR, MR and HR gratings respectively. These values will increase up to $\mathrm{R}\left(\mathrm{EED}_{80}\right) \sim 6,300,11,500$ and 19,500 when using the SCB due to the smaller fiber core. If resolutions are derived from extracted (1D) spectra and are expressed in terms of the FWHM of unresolved spectral lines, then R (FWHM) =6,000, 11,000 and 18,700 for the LCB/MOS and $7,000,12,650$ and 21,500 for the SCB. MEGARA will provide the astronomer unprecedented capabilities to explore the Universe: from the star forming regions, planetary nebulae and massive stars in our galaxy and nearby-galaxies to highredshift clusters of galaxies, being the next workhorse instrument for the GTC scientific community.

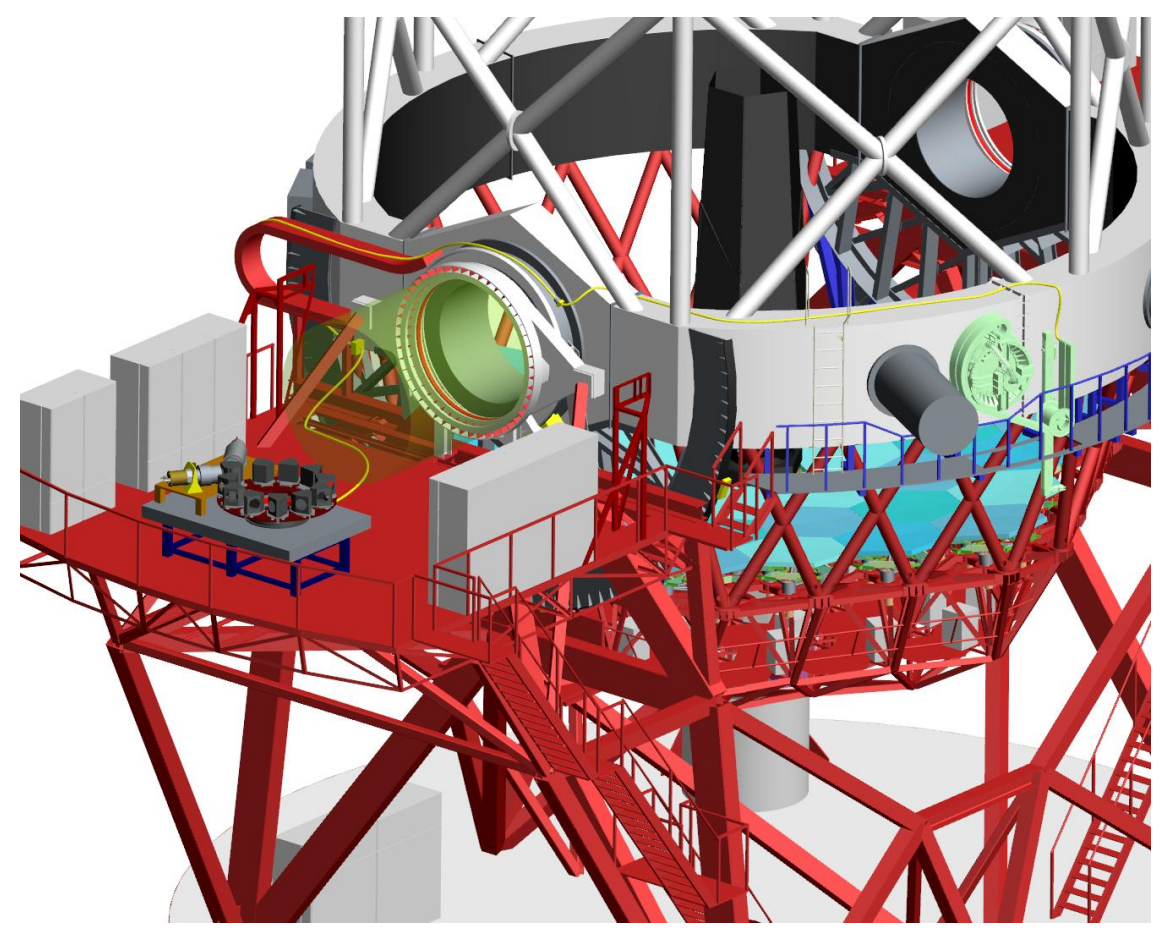

Figure 1. 3D model of MEGARA at the GTC telescope. The Folded-Cass focal station subsystems (in light green at the right of the figure) include the rotator adapter and the interface plates for the Fiber Units (LCB and SCB compact bundles and the positioner robots for the 100 MOS mini-bundles). The bundles are driven from the Folded-Cass to the Nasmyth through the telescope structure and rotators (painted as yellow cables in the figure). Then, the three pseudo-slits (one per bundle) are mounted on a high precision mechanism able to select the bundle in used it and placed it in front of the spectrograph, which remains stable on the platform.

\section{MEGARA OPTICS AT TELESCOPE FOCAL PLANE}

MEGARA Optics at Folded-Cass ${ }^{1,2}$ is composed by: (a) a Field Lens that provides a telecentric focal plane for the microlens arrays (b) the microlenses that change the focal number of the telescope allowing a good coupling with fibers and (c) the fiber bundles themselves. We have paid special attention in designing how the fibers are organized in their route from the telescope Folded Cassegrain focus to the spectrograph entrance and how they are distributed at the pseudo-slit position, which defines the design of the pseudo-slit plates.

\subsection{Overall View of the Fiber Units}

The Fiber Units are composed by the coupling optics, the interface plate where the fibers are mounted, the positioners (in the case of the MOS mode) and the fiber cables, that drive the fiber bundles.

MEGARA has three observing modes, corresponding to the three available fiber bundles: the LCB, the SCB and the MOS. Figure 2 shows how these bundles are placed on the telescope focal plane.

These bundles have 623, 500 and 644 fibers respectively. Fibers are polished, aligned and arranged at both ends of the cable. At the side of the telescope focal plane the fibers are coupled to microlenses to convert the focal ratio of the light 
coming from the telescope (f/17) to that optimal for the optical fibers ( $f / 3)$. The plates where the fibers are mounted will be combined in a larger mechanical interface structure to be attached to the Folded Cassegrain Rotator Adapter. At the other side, the fibers from each bundle have to be arranged in a dedicated pseudo-slit placed the entrance focal plane of the MEGARA spectrograph.

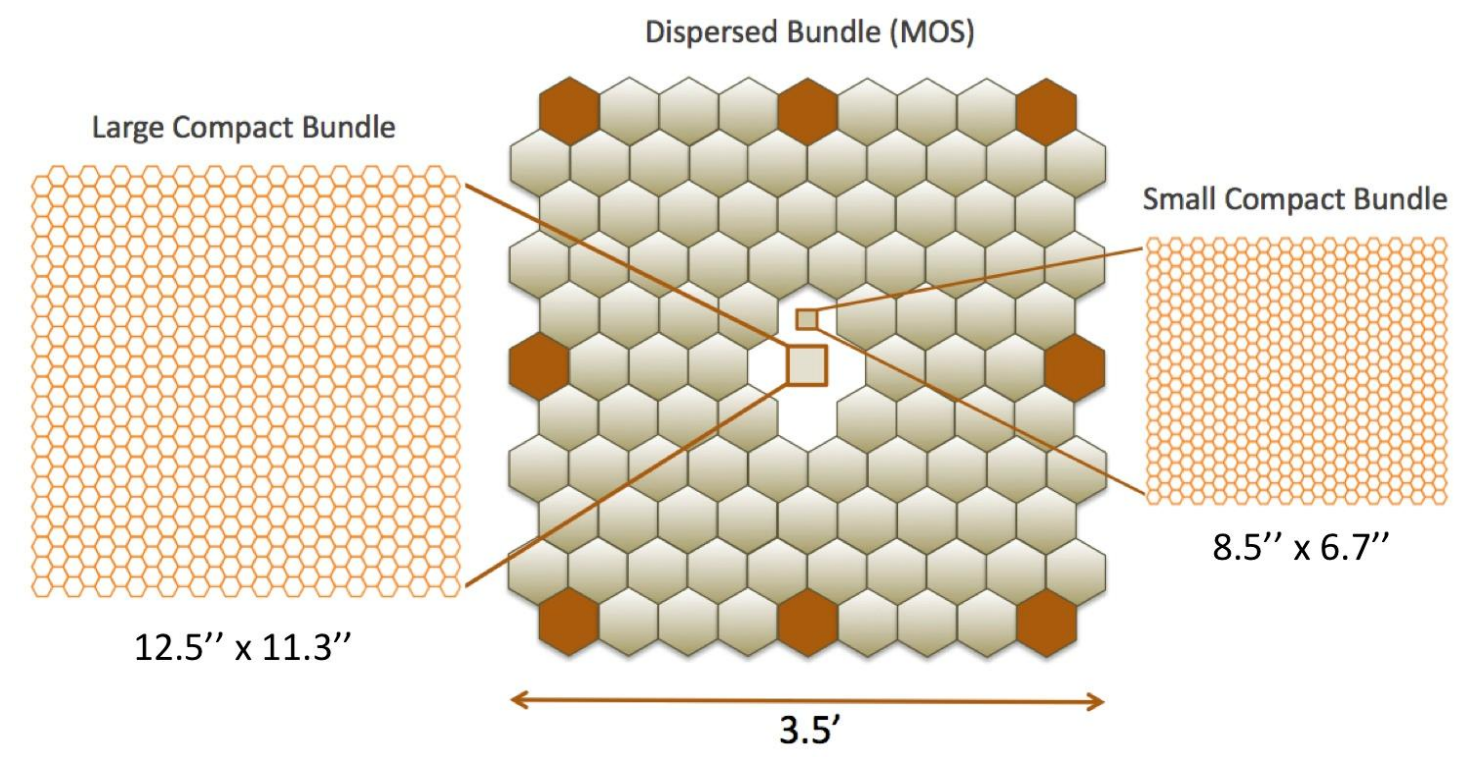

Figure 2. Layout of the Large and Small Compact (LCB and SCB, respectively) and MOS fiber bundles of MEGARA.

A telecentricity corrector is placed in front of the telescope focal plane to guarantee that all microlenses see the same pupil (located at the telescope secondary mirror) and to correct the lack of telecentricity of the MEGARA field of view.

\subsection{Design constrains and high level requirements for the focal plane Optics}

The initial high-level requirements that have been taken into account to study and define the Optics at Folded-Cass are:

The field of view (FOV) has been maximized within the available budget and without impacting appreciably the instrument feasibility. The current configuration has two compact bundles, named LCB and SCB. The LCB covers 12.5 x $11.3 \operatorname{arcsec}^{2}$ on sky (with $100 \mu$-core fibers) centered at the instrument optical axis. The SCB is placed with an offset of $19 \operatorname{arcsec}$ covering a field of $8.5 \times 6.7 \operatorname{arcsec}^{2}$ (with $70 \mu \mathrm{m}$-core fibers). Finally, a system of 100 robotic positioners can place 100 mini-bundles of seven optical fibers each at any place in a $3.5 \times 3.5 \mathrm{arcmin}^{2} \mathrm{FOV}$ around the two IFUs.

The fiber core is critical to define both the resolution element on the sky (called spaxel) and the slit width at the entrance of the spectrograph, which sets the spectral resolution. We adopted a fiber-core diameter of $100 \mu \mathrm{m}$ for the LCB and MOS modes, which corresponds to a projected spaxel size of 0.62 arcsec on sky. This was found to be an appropriate balance between spatial resolution and FOV on one side and spectral resolution and coverage at the detector considering the fiber size - on the other size. For the SCB a fiber-core diameter of $70 \mu \mathrm{m}$ has been adopted, which yields spaxel size of $0.42 \mathrm{arcsec}$. This mode provides both a larger spectral resolution and better spatial sampling that the LCB to the expenses of a more modest field of view. Telecentricity is needed to guarantee that all the microlenses at the focal plane see the same pupil (placed at M2). For this reason a field lens is placed in front of the Folded-Cass focal plane.

The $\mathrm{f} /$ number reduction induced by the microlenses is needed to convert the GTC f/17 beam to a faster one at the entrance of the fibers. We chose $\mathrm{f} / 3$ in order to minimize Focal Ratio Degradation (FRD). The wavelength range spans from 365 to $1000 \mathrm{~nm}$. Coating optimization will be done for the operative range $370-980 \mathrm{~nm}$.

\subsection{Field Lens optical design}

The telescope focal plane is curved. A field lens is designed to provide a telecentric field for MEGARA. With this lens the opto-mechanical axes of all the fiber bundles will be parallel among them. Thus the positioners move on a flat surface (the focal plane) with their opto-mechanical axis perpendicular to this surface. With this solution, we keep a focal 
plane plate scale below $0.1 \operatorname{arcsec}$ in the whole FOV of $3.5 \operatorname{arcmin} \times 3.5 \operatorname{arcmin}$, The lens is a fused silica meniscus (R1= $-2147.6, \mathrm{R} 2=-1731.8 \mathrm{~mm}$ and $\mathrm{CT}=30 \mathrm{~mm}$ ). The optical aperture is $260 \mathrm{~mm}$. The first surface of the lens is placed $68.0 \mathrm{~mm}$ in front of the focal plane in the direction of the optical axis. The field lens position tolerances AOI $<0.01^{\circ}$ guarantee a pupil shift $<1 \mu \mathrm{m}$. As the lens is close to the focal plane it does not change the plate scale. The focal plane position is moved $13.5 \mathrm{~mm}$ from its nominal position (the distance between M2 - and M3 - and the focal plane is increased). The position of the surface of the microlenses is then $\mathrm{z}=-13.5 \pm 0.1 \mathrm{~mm}$ in the GTC F-C coordinate system.

\subsection{Microlens optical design}

The subsystem that introduces the light in the fiber is composed by the microlens arrays and by mechanical supports (that will be a focal plane interface for the LCB and SCB modes and the buttons of the positioners in the case of the MOS mode) where the microlens arrays are glued. The 2D microlens arrays couple the science light at the telescope focal plane into the fibers, re-imaging the telescope pupil on the fiber core. The microlens arrays have the following functions: (a) to define the FOV that will be introduced in the fiber; (b) to adapt the telescope F\# from $\mathrm{f} / 17$ to $\mathrm{f} / 3$ to minimize FRD effects during the beam transport; and (c) to provide a telescope pupil image on the fiber core for stray light control. The microlens arrays will be placed at the telescope focal plane.

Different microlenses (all hexagonal) were designed for different spaxel sizes in order to evaluate the corresponding pupil losses and margins in fiber positioning that would effectively result in negligible fiber-to-fiber variations and, therefore, in a good relative-flux calibration and cosmetics. Regarding the latter issue the spaxel size selected for the LCB/MOS (0.62 arcsec for $100-\mu \mathrm{m}$-core fibers) yields a tolerance in the positioning of the image of the pupil on the fiber core of $10 \mu \mathrm{m}$, which should be fulfilled by the planned centering and gluing procedure. The spaxel size given here is that of the maximum dimension of the hexagonally-shaped microlens (see Figure 3 for the case of LCB and MOS).

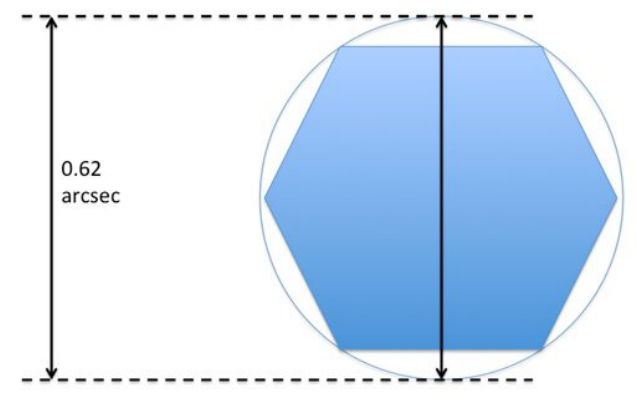

FOV on sky $($ spaxel $)=0.62^{\prime \prime}$

Figure 3. Left, layout of the spaxel size adopted for the $100-\mu \mathrm{m}$-core fibers relative to the circle where it is inscribed. Right, detail of the microlens design (LCB/MOS) showing the telescope focal plane and the FOV aperture.
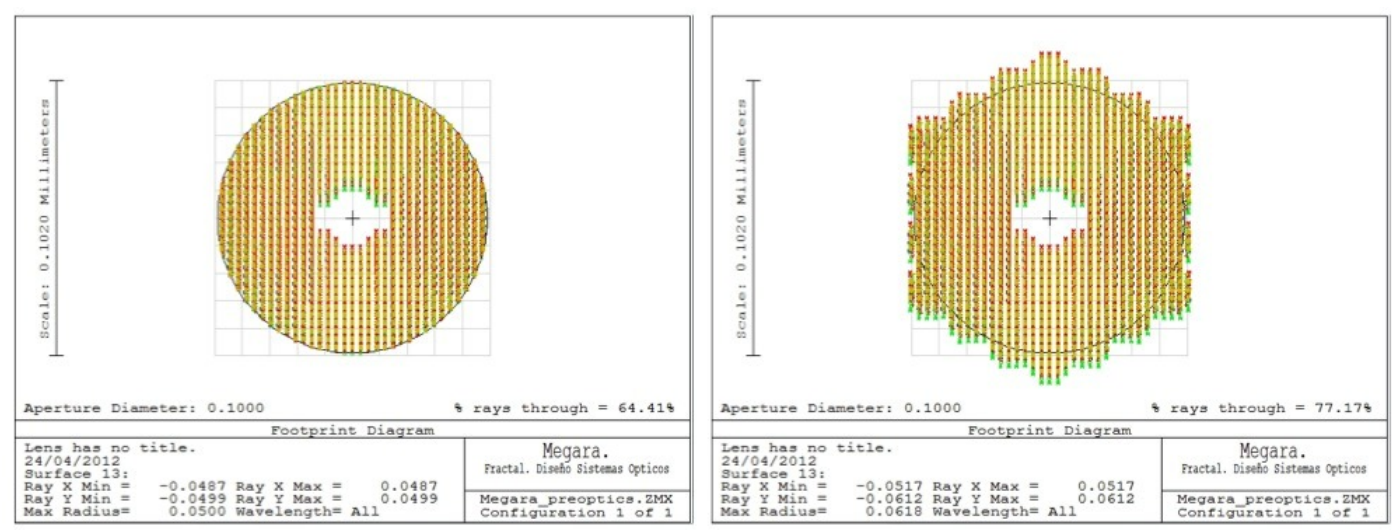

Figure 4. GTC pupil image at the fiber entrance. On the left, the light is entering in the fiber (64.41\% flux). On the right, the complete pupil ( $77.17 \%$ flux) is shown. This is the value corresponding to $100 \%$ of effectiveness. Making the ratio between the two values, we have a transmission of $83.5 \%=64.41 / 77.17$. 
Thus, the total diameter covered by the 7-fiber mini-bundle is $3 x 0.62 x \cos 30^{\circ}=1.61 \mathrm{arcsec}$, in agreement with the value that is required to keep differential Atmospheric Refraction effects under control. The hexagonal microlens inscribed to the circular spaxel guarantees that most of the light will enter the fiber slightly over $\mathrm{f} / 3$ and the losses at the entrance of the spectrograph will be negligible. The oversize of the pupil on the fiber core will decrease $16.5 \%$ the total flux of the maximum telescope aperture, as shown in Figure 4 (for the LCB/MOS microlens). Our design yields a compact ( $100 \%$ filling factor) coverage of the MEGARA IFUs FO. Refractive microlenses are manufactured using a standard semiconductor lithographic technology, allowing very accurate shaping of the lens profile. Table 1 shows the optical properties while Figure 5 shows the performance (also for the LCB/MOS microlens). Similar results have been obtained for the SCB elements. Microlens drawings are complete and the arrays will be manufactured by AMUS Company.

Table 1. MEGARA Microlens optical properties

\begin{tabular}{|l|c|c|}
\hline Property & LCB/MOS microlens & SCB microlens \\
\hline Spaxel on sky / Fiber core & $0.62 / 100 \mu \mathrm{m}$ & $0.42 / 70 \mu \mathrm{m}$ \\
\hline Figure & Aspherical microlens & Aspherical microlens \\
\hline ROC $(\mathrm{mm})$ & 0.844 & 0.563 \\
\hline Thickness $(\mathrm{mm})$ & 2.727 & 1.815 \\
\hline Aperture $(\mu \mathrm{m})$ & 511 & 346 \\
\hline Conic constant & $\mathrm{c}=-0.9797$ & $\mathrm{c}=-0.927$ \\
\hline
\end{tabular}
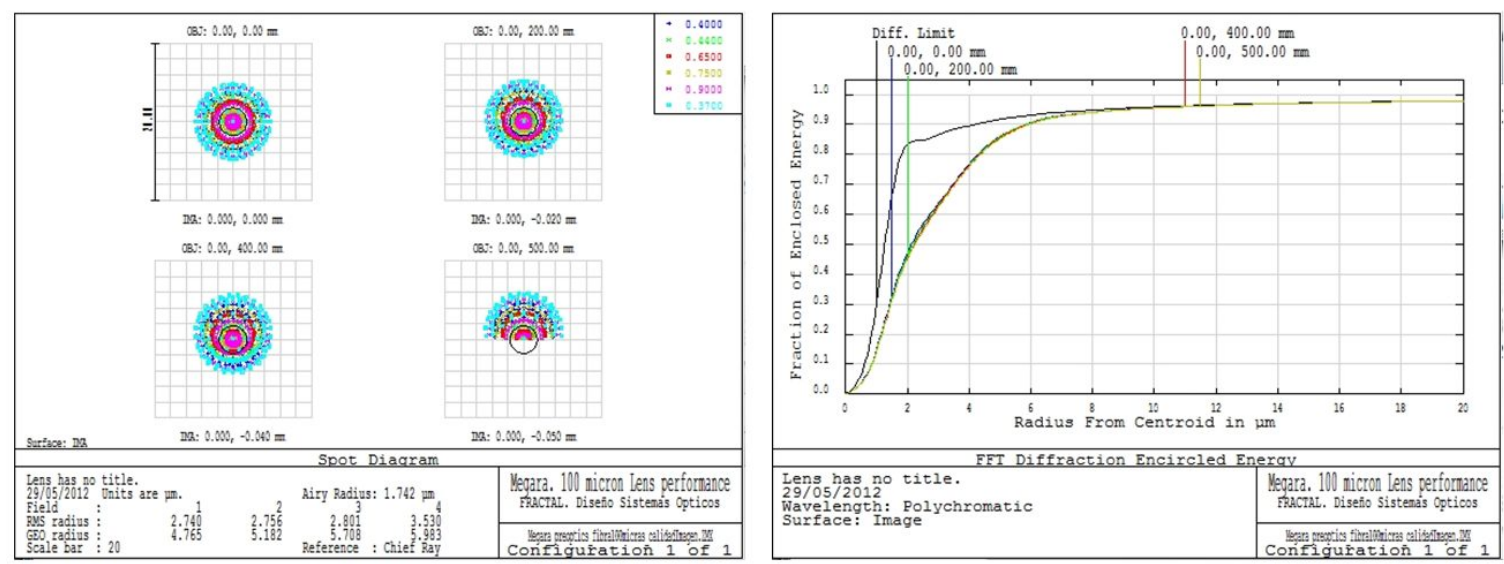

Figure 5. Optical performance of the MEGARA microlenses+field lens in LCB/MOS mode (100 $\mu$ m fiber-core). Left, spot diagram across the fiber core from $0.37 \mu \mathrm{m}$ to $1 \mu \mathrm{m}$. The circle is the Airy pattern at $0.4 \mu \mathrm{m}$. Right, GTC Encircled Energy Diagram (diffraction limited performance shown in black). The image shows the average performance for all wavelengths. No differences between fields are seen.

\subsection{Fiber selection and pseudo-slit arrangement optical design}

The fiber selected for LCB/MOS is the one from Polymicro FBP 100/140/170; $100 \mu \mathrm{m}$ is the core, $140 \mu \mathrm{m}$ is the cladding and $170 \mu \mathrm{m}$ is the mechanical coating. This fiber has a numerical aperture of $0.2 \pm 0.02$ (optical angle acceptance and output light angle of the fiber, sin (12.71)). This is a wide broadband fiber and provides a good FRD. The fibers to be used in the Small Compact Bundle (SCB) share the same manufacturer (Polymicro) and some basic properties with those used for the LCB IFU and MOS modes, such as the numerical aperture and expected transmittance. Besides, we have requested to Polymicro to manufacture the $70 \mu \mathrm{m}$-core fibers with an external mechanical coating of $170 \mu \mathrm{m}$ so to have the same external diameter than in the case of the $100 \mu \mathrm{m}$-core fibers. All fibers will be supported on a mechanical 
interface plate and positioned at the center of the pupil images formed by the microlenses. This plate will be attached to the Folded Cassegrain Rotator Adapter, which will guarantee the position of the fibers in the focal plane. Then, the fibers will transport the science light coupled via the microlenses from this input plane to the spectrograph final position (estimated length of the fiber link is shorter than $40 \mathrm{~m}$ ). We already have a complete mini-bundle with 7 fibers $40 \mathrm{~m}$ each installed at the laboratory where we plan to carry out tests of transmission and FRD before and after gluing the microlens arrays. Also, in order to have an accurate measure of the fiber link length, we plan to install in the GTC a cover tube (same as the one to be used to group the fiber bundles) but with no fibers inside between the MEGARA Folded Cassegrain focus and the spectrograph entrance. Fiber bundles shall be manufactured by SEDI Company.

The LCB IFU fibers shall be all grouped in a single large tube, which shall have octopus geometry and shall be divided into small sub-units where the fibers coming to each pseudo-slit box shall be included. All the MOS units and the 8 fixed minibundles shall be integrated in sub-units of 7 fibers. Finally, the SCB fibers must be also arranged in an octopus geometry tube as in the LCB. Each fiber bundle (LCB, MOS, SCB) shall be independently covered by polyurethane loose tubing (not a tight jacket). A minimum bend radius of $50 \mathrm{~mm}$ shall be respected. In order to avoid different radii of curvature between the outer and inner fibers in each bundle, the fibers inside a fiber bundle shall be braided.

\section{MEGARA SPECTROGRAPH OPTICS DESCRIPTION}

\subsection{Requirements rationale for the spectrograph optical design}

MEGARA has been designed based on a clear set of scientific requirements summarized in Table 2.

Table 2. MEGARA requirements for the optical design

\begin{tabular}{|l|c|}
\hline Requirement & Value \\
\hline Wavelength range & $3700-9800 \AA$ \\
\hline Fiber core for LCB and MOS & $100 \mu \mathrm{m}$ \\
\hline Fiber core for SCB & $70 \mu \mathrm{m}$ \\
\hline Detector pixel size & $15 \mu \mathrm{m} / \mathrm{pix}$ \\
\hline Detector format & 4096 pix x 4096 pix \\
\hline F number at the fiber exit & $\mathrm{f} / 3$ \\
\hline Spectral Resolution Element (LCB/MOS) & 4 pixels \\
\hline Spectral resolutions (EED 80 for LCB/MOS & $5500,10000,17000$ \\
\hline Spectral Resolution Element (SCB) & $\sim 3.5$ pixels \\
\hline Spectral resolutions $\left(\mathrm{EED}_{80}\right.$ ) for SCB/MOS & $6300,11500,19500$ \\
\hline Fiber-Fiber separation on detector & 2 pixels $( \pm 10 \%)$ \\
\hline
\end{tabular}

The rationale behind these requirements is explained as follows:

- Wavelength range: the spectrograph will have a single arm (the whole range will be observed with the same Optics). This implies strong constraints in the optical design but it is a need due to the small instrument budget.

- Plate scale: The plate scale sets the fcoll/fcam ratio. As 0.620 " has been selected on the sky and the spectral element is required to be sampled with 4 pixels, we have considered the 0.620 "projected in 3.33 pixels giving a 
final plate scale of $0.186 \%$ pixel on the detector. The difference between 3.3 and 4 pixels is available for image quality error budget.

- Scale Reduction factor: We will project the fiber size core $(100 \mu \mathrm{m})$ in 3.33 pixels by design. As pixel size is $15 \mu \mathrm{m}$ wide, 3.3 pixels are $50 \mu \mathrm{m}$, what implies a scale reduction between the telescope focal plane and the detector of a factor of 2 . This factor will fix the ratio between the collimator and camera $\mathrm{f} /$ numbers.

- Collimator and camera $\mathbf{f}$ numbers: The spectrograph entrance $\mathrm{f} /$ number is chosen to minimize the FRD of the fiber link, thus $f / 3$ is used at the entrance and exit of the fiber becoming the Fcoll\#. Since the focal ratio Fcoll/Fcam is 2 then average values for $\mathrm{F} \#$ collimator $=3$ and for $\mathrm{F} \#$ camera $=1.5$ (what is feasible). Faster (smaller) f/ numbers for the camera become very difficult to achieve for such a large and complex camera.

- Pupil size: The FOV will limit the minimum pupil size. As we move to wider FOV the total focal length of the collimator and camera has to be increased in order to maintain the image quality. With the current experience filling $4 \mathrm{~K}$ pixels in spatial direction will require a minimum of $160 \mathrm{~mm}$ pupil in order to manage field aberrations. This size (Ø160mm pupil) has been fixed and will limit the maximum (pseudo-slit) length.

- Spectral resolution: The linear spectral resolution is proportional to the camera focal length. Thus using a smaller pupil will shorten the focal length and the linear dispersion at the end. For example, using a half diameter pupil would decrease the resolution to half of the value if we maintain the four pixels per resolution element. In order to recover the nominal resolution two options were explored at the Feasibility Study Phase: (a) doubling the angular dispersion of the dispersion element. This can be done at $\mathrm{R}=5,500$ but not at $\mathrm{R}=17,000$ as we are in the limit of the geometry, so it is not an option if we are required to maintain HR and (b) decreasing the fiber size to half of the value (thus $50 \mu \mathrm{m}$ ) and using two pixels per resolution element (not acceptable due to the spaxel size scientific requirement). These two solutions were discarded early on in the MEGARA design.

- Spectral resolution and geometry: The grating equation for the angular dispersion sets the geometry of the light angle of incidence on the grating. This equation combines the wavelength $(\lambda)$; the Angle Of Incident (AOI) on the grating, $\beta$, the number of lines $/ \mathrm{mm}$ ruled on the grating $(\mathrm{m})$ and the diffraction order $(\mathrm{n})$. The best VPH performance regarding efficiency is normally obtained at first order $n=1$. This means that setting a HR mode will require a high AOI on the grating, while LR mode requires a low AOI on the grating. Different solutions can be offered for fitting a wide range of spectral resolutions: (a) to consider two spectrographs geometries (everything is the same except the envelope and the fixed geometry). This means to have two different spectrographs, one specific for HR and the other one for LR. This is optimum in terms of optical design; (b) to have a single spectrograph with an articulated camera-grating that changes the geometry. In this case, the instrument would require a higher envelope and motorization of the grating and the camera-detector stage, what is not desirable and produced undesirable calibration problems or (c) a single spectrograph with a fixed geometry between the HR and the LR optimum angles. The final geometry on the grating is obtained by sandwiching the gratings between two prisms, what will change the beam's angle to the required one.

All these previous options share the same optical design regarding the collimator and camera design. However, some differences are found regarding the gratings. A detailed trade-off analysis showing the possible alternatives to widen the spectral resolution range and/or the wavelength coverage at the longest wavelengths was done and it was decided to close the MEGARA Optics concept with a fixed angle between collimator and camera. Therefore the possibility of using an articulated camera was definitively ruled out. We concluded that option (c) is the best one but even in this case we included a novel design for the highest resolution mode based on sliced pupil gratings ${ }^{5,6}$. An alternative to the sliced-pupil grating, using a decker with a single prism will also be studied during Detailed Design and a final solution will be closed after the Critical Design Review (CDR).

- Spectral elements: The VPHs are proposed as the spectral dispersers of the instrument. Ruled gratings are not available with the number of lines $/ \mathrm{mm}$ and are not so efficient. Moreover transmission gratings are preferred over reflective ones because of the smaller size of the camera optics, as because in this case camera elements can be located very close to the dispersive element. In the VPHs, the index pattern (hologram) is photo-recorded in dichromate gelatin. The hologram parameters (lines $/ \mathrm{mm}$, AOI on the grating gives the wavelength that meets the Bragg condition). These values provide the required resolutions for the proposed gratings. The hologram 
thickness can be tuned to avoid a high dependence in the efficiency when out of Littrow angle. The AOI within the gelatin is given by the grating equation (see equation 1) where $m=1$ is the order, $\lambda$ is the Bragg wavelength, $\mathrm{d}$ is the grating spacing, and $\mathrm{n}=1.27$ is the gelatin index.

$$
2 \mathrm{dn} \sin \beta_{\mathrm{m}}=\mathrm{m} \lambda
$$

The overall geometry implies that the required working angles will be around $26^{\circ}$ in the LR, around $44^{\circ}$ in the MR and around $70^{\circ}$ in the HR. In order to change the AOI from a fixed geometry to the different angles required, prisms are used. The grating will be sandwiched inside. The prism material will depend on the grating AOI. When the light goes from the gelatin to the material substrate or vice versa, we apply the Snell law.

$$
\mathrm{n}_{\text {gelatin }} \sin \beta=\mathrm{n}_{\text {prism }} \sin \alpha
$$

- Spectral resolution and fiber size: Changing the spectral resolution can also be done by using different fiber sizes. For example the use of $100 \mu \mathrm{m}$ fiber core for $\mathrm{R}=10,000$ (projecting 1 fiber on 4 pixels) and $50 \mu \mathrm{m}$ fiber core for $\mathrm{R}=20,000$ (projecting 1 fiber on 2 pixels) provides both resolutions with the same diffraction element. We discarded this solution for MEGARA because, on the one hand, it is desirable to use the same type of fibers to have the same spaxel on sky with all the resolutions and, on the other hand, because the plate scale on sky covered by a $50 \mu \mathrm{m}$ fiber is very small $(0.3$ arcsec $)$ implying a sub-seeing sampling and a reduced FOV. MEGARA uses an additional bundle, SCB, of 500 fibers and $70 \mu \mathrm{m}$ fiber core to reach a higher regime of spectral (and spatial) resolutions with the same VPH units than using with the LCB/MOS bundles.

The final optical design configuration is composed by a collimator and a camera keeping a fixed angle of $68^{\circ}$. The pupil size is $160 \mathrm{~mm}$ and the gratings (based on VPHs) will be located at pupil.

\subsection{Collimator}

The collimator is composed by a singlet and two doublets (see Figure 6). The average paraxial focal length is $483.3 \mathrm{~mm}$ and it is a refractive system with $\mathrm{F} \# 3$. The specifications and manufacturing tolerances are summarized in Table 3.

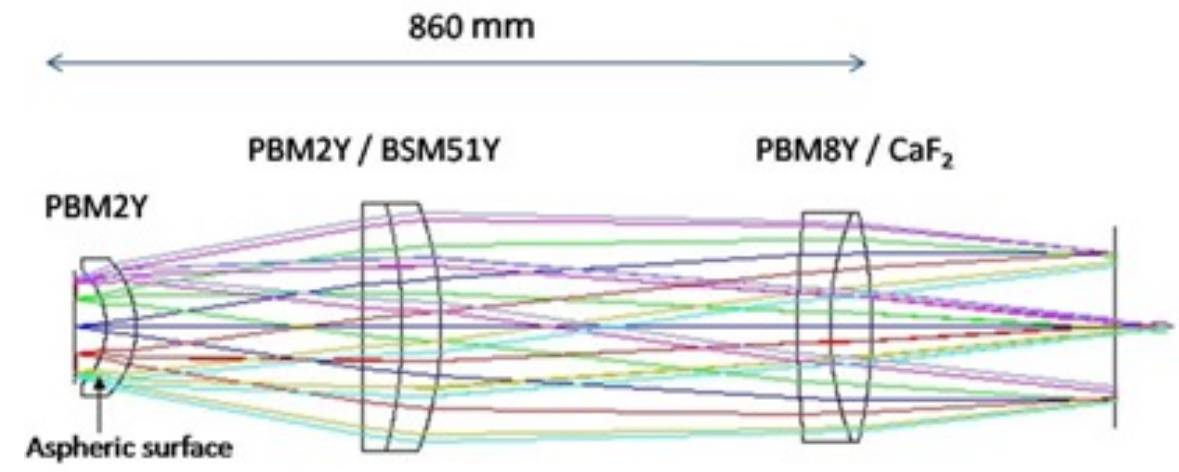

Figure 6. Collimator layout. It is composed by one PMB2Y singlet (aspherical) and two doublets.

Table 3. Collimator lens parameters and manufacturing tolerances. D2/D3 and D4/ D5 are cemented in doublets.

\begin{tabular}{|c|c|c|c|c|c|c|}
\hline Element & $\mathrm{R} 1(\mathrm{~mm})$ & $\mathrm{R} 2(\mathrm{~mm})$ & $\begin{array}{c}\text { Central } \\
\text { Thickness }(\mathrm{mm})\end{array}$ & $\begin{array}{c}\text { Wedge } \\
\text { (arcmin) }\end{array}$ & $\begin{array}{c}\text { Surf } \\
\text { (fringes @ } \\
0.632 \mathrm{~nm})\end{array}$ & $\begin{array}{c}\text { Index } \\
\text { tolerance }\end{array}$ \\
\hline COLL S-1 & $-97.0 \pm 0.1$ & $-113.3 \pm 0.1$ & $35.00 \pm 0.15$ & \pm 2 & - & $\pm 10^{-4}$ \\
\hline COLL D-2 & flat & $-728.1 \pm 1.0$ & $35.00 \pm 0.15$ & \pm 2 & 2 & $\pm 10^{-4}$ \\
\hline COLL D-3 & $-728.1 \pm 1$ & $-398.8 \pm 0.4$ & $35.00 \pm 0.15$ & \pm 2 & 2 & $\pm 10^{-4}$ \\
\hline COLL D-4 & $1259.9 \pm 2$ & $344.5 \pm 0.5$ & $25.00 \pm 0.15$ & \pm 2 & 2 & $\pm 10^{-4}$ \\
\hline COLL D-5 & $344.5 \pm 0.5$ & $-542.5 \pm 0.5$ & $45.00 \pm 0.15$ & \pm 2 & 2 & $\pm 10^{-4}$ \\
\hline
\end{tabular}


The lens COLL-S1 element is a high-order aspheric (Figure 7). The aspheric coefficients are $-2.611 \times 10^{-8} \pm 7.7 \times 10^{-10}$ for $\mathrm{R}^{4}$ and $-1.600 \times 10^{-11} \pm 2.14 \times 10^{-13}$ for $\mathrm{R}^{6}$.

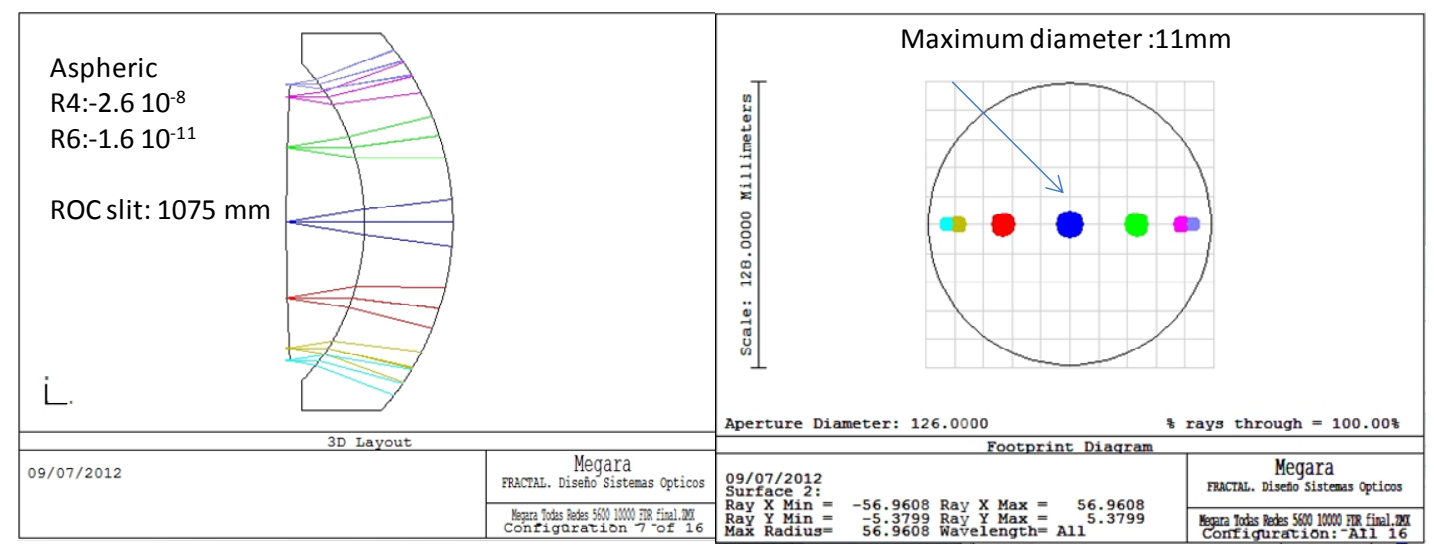

Figure 7: The Collimator S1 element is a high order aspheric. Figure shows optical parameters and footprint diagram.

\subsection{Camera}

The camera is composed by two doublets and 3 singlets as seen in Figure 8 . The average paraxial focal length is $245.9 \mathrm{~mm}$ and it is a refractive system with $\mathrm{F} \# 1.5$. The image field is $61.4 \mathrm{~mm} \times 61.4 \mathrm{~mm}$ covering $4 \mathrm{~K}$ x $4 \mathrm{~K}$ pixels. The optical elements specifications and manufacturing tolerances are summarized in Table 4.

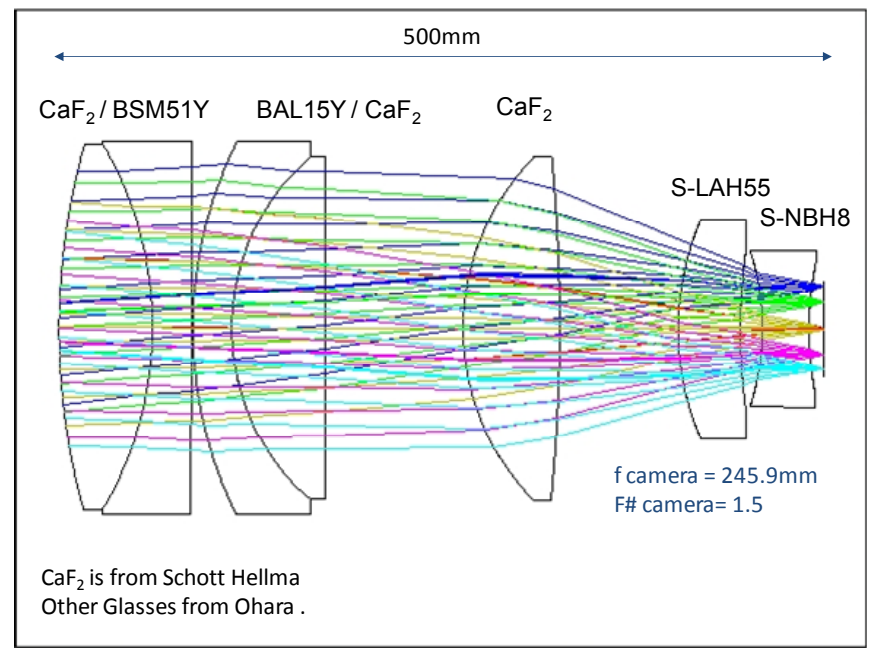

Figure 8: Camera layout. It is composed by two doublets, each of them with one $\mathrm{CaF}_{2}$ lens and materials as labeled in the figure, and three singlets of $\mathrm{CaF}_{2}, \mathrm{~S}-\mathrm{LAH} 55$ and S-NBH8, respectively. The last lens acts also as cryostat window.

Table 4. Camera lens parameters and manufacturing tolerances. D1/D2 and D3/D4 are cemented in doublets.

\begin{tabular}{|c|c|c|c|c|c|c|}
\hline Element & R1 $(\mathrm{mm})$ & $\mathrm{R} 2(\mathrm{~mm})$ & Thickness & $\begin{array}{c}\text { Wedge } \\
(\operatorname{arcmin})\end{array}$ & $\begin{array}{c}\text { Surf (fringes } \\
@ 0.632 \mathrm{~nm})\end{array}$ & Index tolerance \\
\hline CAM D-1 & $435.9 \pm 0.4$ & $-231.7 \pm 0.2$ & $60.0 \pm 0.1$ & \pm 2 & 2 & $\pm 10^{-4}$ \\
\hline CAM D-2 & $-231.7 \pm 0.2$ & Flat & $25.0 \pm 0.1$ & \pm 2 & 2 & $\pm 10^{-4}$ \\
\hline CAM D-3 & $269.2 \pm 0.2$ & $145.1 \pm 0.1$ & $25.0 \pm 0.1$ & \pm 2 & 2 & $\pm 10^{-4}$ \\
\hline CAM D-4 & $145.1 \pm 0.1$ & Flat & $60.0 \pm 0.1$ & \pm 2 & 2 & $\pm 10^{-4}$ \\
\hline CAM S-5 & $156.0 \pm 0.1$ & $-1143.0 \pm 0.8$ & $62.0 \pm 0.1$ & \pm 2 & 2 & $\pm 10^{-4}$ \\
\hline CAM S-6 & $176.4 \pm 0.2$ & $365.8 \pm 0.3$ & $40 \pm 0.1$ & \pm 2 & 2 & $\pm 10^{-4}$ \\
\hline CAM S-7 & $-162.5 \pm 0.2$ & $219.5 \pm 0.2$ & $30 \pm 0.1$ & \pm 2 & 2 & $\pm 10^{-4}$ \\
\hline
\end{tabular}


We have evaluated the thermal effects affecting image quality. The nominal model at $20^{\circ} \mathrm{C}$ at $\mathrm{P}=1 \mathrm{~atm}$ was moved at $\mathrm{T}=$ $-4^{\circ} \mathrm{C}$ and $\mathrm{T}=+20^{\circ} \mathrm{C}$ with $\mathrm{P}=0.77 \mathrm{~atm}$. The camera turned to be the worst offender and has been passively athermalized.

The minimum distance between the last surface of the last camera lens (which is the cryostat window) and the detector at the edge is $4.7 \mathrm{~mm}$. The detector plane is tilted by and angle of $-0.136^{\circ}$ (kept by a shim at one side).

\subsection{Pupil Elements}

Different types of pupil elements (all of them based on VPH-type gratings) can be accommodated in the pupil position to provide the different spectral modes, with resolution power of 5,500 (LR), 10,000 (MR) and 17,000, when using in the $\mathrm{LCB} / \mathrm{MOS}$ mode. These values are referred to $\mathrm{EED}_{80}$. A maximum of $20 \%$ of different resolution is allowed due to the angle of $7.2^{\circ}$ to see the whole camera. LR units are built with simple gratings sandwich between two flat windows; MR units are provided with the gratings sandwiched between two symmetric prisms that allow the beam to incident on the VPH in the optimum angle. Finally, HR units shall be designed as sliced-pupil gratings or as single-slice ones combined with a decker. A summary of the optical parameters of the VPH units is shown in Table 5. For the reddest gratings, an order sorting filter will be used (placed at the shutter position).

Table 5: MEGARA Baseline gratings: VPH specifications.

\begin{tabular}{|c|c|c|c|c|c|c|}
\hline VPH Name & $R_{\text {EED80 }}$ & $\begin{array}{c}\lambda \text { Bragg } \\
\AA\end{array}$ & $\begin{array}{c}\theta \text { Bragg } \\
\text { degrees }\end{array}$ & $\begin{array}{c}\text { Record } \\
\text { lines/mm }\end{array}$ & $\begin{array}{c}\text { Prism Apex } \\
\text { degrees }\end{array}$ & $\begin{array}{c}\text { Optical Aperture } \\
\mathrm{mm} \times \mathrm{mm}\end{array}$ \\
\hline VPH405-LR & 5,473 & 4,051 & 26.12 & 2,761 & Flat & $172 \times 200$ \\
\hline VPH480-LR & 5,504 & 4,800 & 26.12 & 2,330 & Flat & $172 \times 200$ \\
\hline VPH570-LR & 5,523 & 5,700 & 26.12 & 1,962 & Flat & $172 \times 200$ \\
\hline VPH675-LR & 5,533 & 6,749 & 26.12 & 1,657 & Flat & $172 \times 200$ \\
\hline VPH865-LR & 5,559 & 8,650 & 26.12 & 1,293 & Flat & $172 \times 200$ \\
\hline VPH410-MR & 11,074 & 4,104 & 51.65 & 4,854 & 44.00 & $172 \times 205$ \\
\hline VPH443-MR & 11,053 & 4,431 & 51.88 & 4,510 & 44.00 & $172 \times 205$ \\
\hline VPH481-MR & 10,980 & 4,814 & 51.86 & 4,150 & 44.00 & $172 \times 205$ \\
\hline VPH521-MR & 10,930 & 5,213 & 51.85 & 3,832 & 44.00 & $172 \times 205$ \\
\hline VPH567-MR & 10,870 & 5,667 & 51.61 & 3,513 & 44.00 & $172 \times 205$ \\
\hline VPH617-MR & 10,830 & 6,170 & 51.39 & 3,217 & 44.00 & $172 \times 205$ \\
\hline VPH712-MR & 10,660 & 7,115 & 51.09 & 2,778 & 44.00 & $172 \times 205$ \\
\hline VPH777-MR & 10,660 & 7,767 & 50.96 & 2,540 & 44.00 & $172 \times 205$ \\
\hline VPH926-MR & 10,500 & 9,262 & 50.73 & 2,123 & 42.00 & $172 \times 205$ \\
\hline VPH665-HR & 17,000 & 6,646 & 70,02 & 3,592 & N/A & $172 \times 230$ \\
\hline VPH863-HR & 17,000 & 8,634 & 69,77 & 2,761 & N/A & $172 \times 230$ \\
\hline
\end{tabular}

A mechanism (linear stage) at the pseudo-slit allows fine focusing for improving image quality in the different grating configurations. This system adjusts the distance between the pseudo-slit plane and the first surface of COLL-S1. The focus depth implies that the minimum step is $10 \mu \mathrm{m}$ and the total range should be $\pm 3 \mathrm{~mm}$.

\section{MEGARA OPTICS PERFORMANCE}

\subsection{Transmission}

We have estimated the total transmission for MEGARA taking into account both, Folded-Cass (FC) optics and spectrograph. The total FC subsystems transmission has been evaluated for LCB/MOS and SCB modes separately. Figure 9 shows the result for LCB and MOS modes. A similar result is obtained for SCB mode. This estimation includes the following contributors (we indicate also the hypothesis considered). In the case of the F/C optics we assume:

- Field lens includes Fresnel losses face (1.5\% loss per surface - 2 surfaces). Fused Silica losses are negligible.

- Microlens includes Fresnel losses due to microlens AR coating (1.5\% per one surface) and a maximum of 5\% loss of the array area due to the boundary effect between lenslets. 
- We have included the losses due to the pupil oversize on the LCB/MOS fiber core in order to ensure the flux calibration. This issue will decrease $16.5 \%$ the total flux of the maximum telescope aperture in the case of the $100 \mu \mathrm{m}$ fiber-core (LCB and MOS modes). In the case of the $70 \mu \mathrm{m}$ fiber-core SCB mode this number is $8.8 \%$.

- Fiber transmission is computed taken into account the attenuation $(\mathrm{db} / \mathrm{km})$ reported by Polymicro for each wavelength and considering a fiber length of $40 \mathrm{~m}$ (distance between the FC station and the Nasmyth platform, where the spectrograph will be located). Regarding FRD, according to the figures provided by Polymicro, the loss can be between $2 \%$ and $5 \%$. We have adopted an average value of $3.5 \%$. Finally, a loss of $1.5 \%$ (Fresnel) at the fiber exit has been included. Fiber transmission and FDR shall be measured at laboratory.

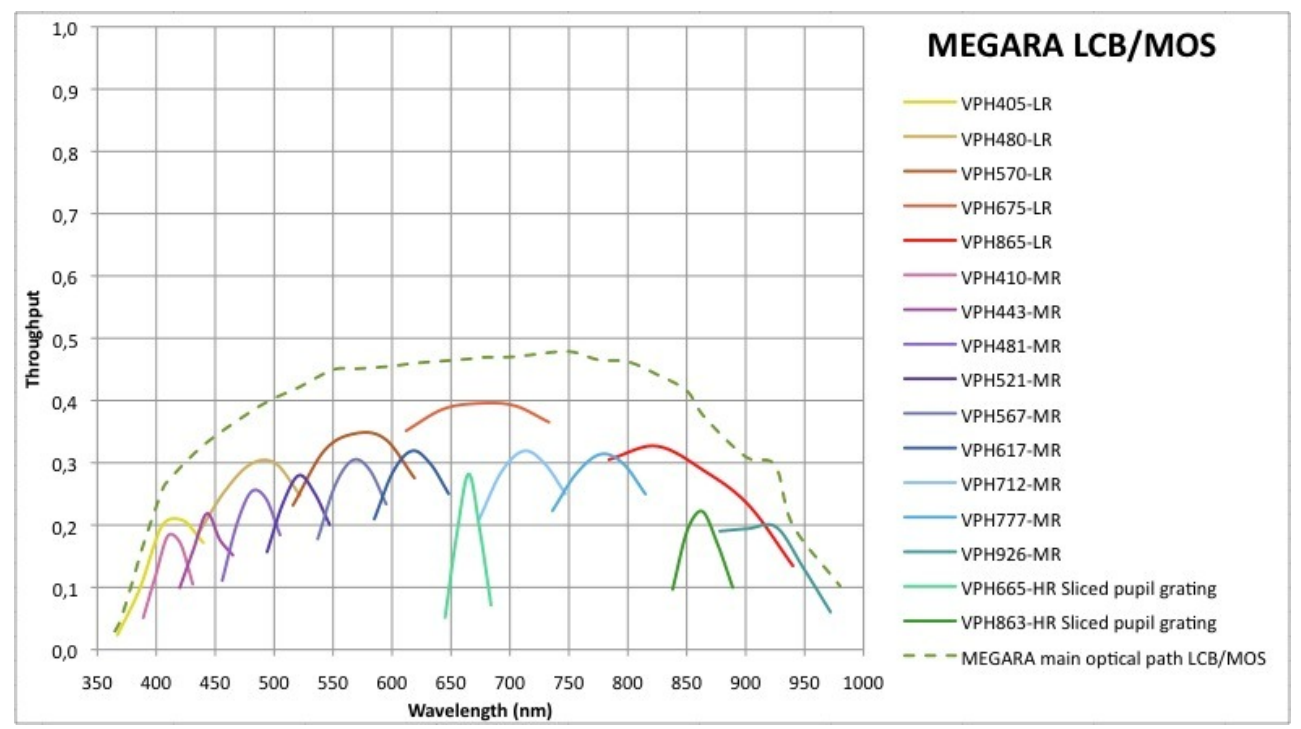

Figure 9: Total transmission provided by MEGARA for LCB and MOS modes evaluated at the different VPH gratings.

Regarding spectrograph optics, we have included the following assumptions in the transmission estimation:

- Main Optics is composed by the collimator and the camera (12 lenses). We have assumed 1.5\% Fresnel losses per glass/air interfaces (a worse case since INAOE and CIO are working to provide coatings better than 1.3\%).

- Detector includes the Quantum efficiency of the CCD231-E74 (layer Astro AR Mult-2) as provided by e2V.

- All VPH gratings are evaluated in five points: at the edges of the wavelength range provided by each grating, at the center wavelength and at other two wavelength values in the middle between the center and the edges.

- VPH efficiency values are the ones provided by the manufacturer and includes $6 \%$ margin (for scattering and hologram defects). In our experience this margin can be reduced to $1 \%-2 \%$ in the real elements.

- LR and MR gratings transmission estimation includes both the VPH efficiency and the effects at the interfaces between the components sandwiching the VPH. For LR units, window material (Fused Silica) losses are negligible. We include the air-window interface ( $1.5 \%$ per surface -2 surfaces) and the window-gelatin interface ( $1 \%$ per surface -2 surfaces). For MR units, material (PBM2Y) losses are estimated less than $1 \%$. The interface losses include the air-prism interface (1\% per surface due to short wavelength range -2 surfaces) and at the prism-gelatin interface ( $1 \%$ per surface -2 surfaces). As far as the gratings will be used in a fixed angle, optimized coatings can be obtained for each wavelength range (currently under study by INAOE).

- Order sorting filter loss is estimated in $1.5 \%$ and has been included together with the grating when needed.

- HR sliced-pupil grating includes the overall high resolution grating efficiency taking into account together the VPH efficiency, the losses at the interfaces between the optical components that sandwiched the VPH in the case of the high resolution sliced pupil gratings and the vignetting due to divide the pupil. Material (Fused silica) losses are negligible and PBM2Y losses are estimated to be less than $1 \%$. The interface losses include the air-prism interface ( $1 \%$ per surface due to short wavelength -2 surfaces), at the prism-NOA61 glue ( $1 \%$ per surface -2 surfaces) and at the window-gelatin interface ( $1 \%$ per surface -2 surface). The losses at the 
interface between glue and window are negligible because their similar refractive indices. In addition, due to the high AOI in the HR gratings, it is possible that the losses at the interfaces could slightly degrade at the edges. This is a very small effect that will be minimized by optimizing the AR coating. Finally, the vignetting coming from the sliced-pupil solution and the variations between the center and the edge have both been included.

\subsection{Image Quality and associated Error Budget}

The image quality requirement is to have the resolution element in four pixels $(60 \mu \mathrm{m})$. The value that contains the $80 \%$ of the encircled energy coming from a fiber whose projection is $50 \mu \mathrm{m}$ is a diameter of $34.32 \mu \mathrm{m}$ (equation 3 ).

$$
\mathrm{EE}_{80}=60^{2}-34.32^{2}=49.2^{2}
$$

For this reason the total value of the $\mathrm{EE}_{80}$ through the optical system has to be lower or equal to $49.2 \mu \mathrm{m}$ in diameter ( $24.6 \mu \mathrm{m}$ radius). The value of the requirement when analyzing the performance will have a value of $\mathrm{EER}_{80} \leq 24.6 \mu \mathrm{m}$.

For practical purposes (optical analysis) the evaluation of the Error Budget (EB) will not be made in terms of $\mathrm{EED}_{80}$, but in terms of RMS spot radius, and considering 1D Gaussian profile in the spectral direction. Thus, in order to compute the EB, we will use the value of the $\sigma$ of the Gaussian corresponding to the $80 \%$ of the energy, related as in equation (4).

$$
\sigma=\mathrm{EER}_{80} / 1.28=19.22 \mu \mathrm{m}
$$

Table 6 summarizes the EB contributors. The errors associated to different tolerances were computed analytically or with MC models. In the case of MC models a normal probabilistic distribution between the lower and upper limits was used and the associated error was considered at the $90 \%$, which means that $90 \%$ of the systems would be within expectations.

Table 6: Estimated values for the different Image Quality EB contributors

\begin{tabular}{|l|c|l|}
\hline \multicolumn{1}{|c|}{ ITEM } & $\begin{array}{c}\sigma(\mu \mathrm{m}) \\
100 \mu \mathrm{m} \text { fiber core }\end{array}$ & \multicolumn{1}{|c|}{ Comment } \\
\hline Nominal performance & 8.50 & Nominal design in one representative mode \\
\hline $\begin{array}{l}\text { Collimator fabrication (lens thickness, } \\
\text { wedge, surface irregularity, curvature) }\end{array}$ & 2.26 & 200 Monte Carlo runs in normal distribution \\
\hline $\begin{array}{l}\text { Camera fabrication (lens thickness, } \\
\text { wedge, surface irregularity, curvature) }\end{array}$ & 2.96 & 200 Monte Carlo runs in normal distribution \\
\hline $\begin{array}{l}\text { Collimator AIV (axial and lateral } \\
\text { decentering, tilts). }\end{array}$ & 1.95 & 200 Monte Carlo runs in normal distribution \\
\hline $\begin{array}{l}\text { Camera AIV (axial and lateral } \\
\text { decentering, tilts). }\end{array}$ & 3.10 & 200 Monte Carlo runs in normal distribution \\
\hline Uncompensated & 3.60 & 200 Monte Carlo runs in normal distribution \\
\hline Thermal & 2.10 & Worst case. Analytical model \\
\hline Glass homogeneity & 3.60 & Analytical model \\
\hline Pupil elements & 5.00 & Allocated Estimation \\
\hline Detector Flatness & 1.32 & Considering flatness \pm 5 $\mu \mathrm{m}$ \\
\hline Pseudo-slit curvature & 3.20 & \\
\hline Atmospheric Effect & 0.14 & Performance Difference between 0.77-1atm \\
\hline TOTAL (rms squared) & 12.92 & \\
\hline
\end{tabular}


The EB has to include all the contributors to image quality, whose quadratic sum has to reconstruct the requirement as:

$$
\sigma_{\text {total }}^{2}=\sigma_{\text {design }}^{2}+\sigma_{\text {manufacturing }}^{2}+\sigma_{\text {alignment }}^{2}+\sigma_{\text {thermal }}^{2}+\ldots
$$

The values for the nominal design evaluation have been obtained from the grating VPH481-MR, like an average of the image quality values at different wavelengths $(457,470,481,492$ and $503 \mathrm{~nm})$ and field positions along the pseudo-slit $(0, \pm 30$ and $\pm 55 \mathrm{~mm}$ ). The average rms distance in the $\mathrm{Y}$ (spectral axis) corresponds to $\sigma \leq 8.5 \mu \mathrm{m}$. We note that the pupil-elements contribution quoted in Table 6 is probably overestimated since passive compensation could be used if needed. Also, the value for the pseudo-slit curvature effect is considering only one grating. The average value when all VPH gratings are considered is $3.7 \mu \mathrm{m}$. Finally, the maximum error in detector flatness is $\pm 5 \mu \mathrm{m}$ and was evaluated considering a mean focus shift of $\pm 5 \mu \mathrm{m}$ without further compensation. This gives a degradation of $1.32 \mu \mathrm{m}$.

The expected result once the instrument will be built according to this EB is $\sigma=12.92 \mu \mathrm{m}$, while the total value can be up to $\sigma=19.22 \mu \mathrm{m}$. There is room for improvement that will be used to fit the 3 pixels requirement in the case of the SCB.

Manufacturing errors: The tolerance parameters are radius of curvature, lens thickness, wedge and the aspheric parameters (see tables 3 and 4). We consider a tolerance of $\pm 10^{-4}$ for the refraction index corresponding to the variation within a lot of fine annealed glass. Abbe tolerance effect was negligible and thus not considered. The surface irregularity was calculated following the Zemax model that is giving in fringes the total irregularity at the maximum lens aperture and splits it between astigmatism and spherical at 50\%. For all the cemented doublets only the external faces are considered, as the coupling materials makes the contribution to the wavefront error of these surfaces much smaller for the same quality. A sensibility analysis of the design was made for the collimator and camera in order to identify the critical parameters. The model evaluates the image quality up to the focal plane position. From the sensitivity analysis we obtained the compensators for each unit that will be assembled independently.

Regarding the collimator, we propose manufacturing all lenses in a single run with the tolerances given in Table 3 . The compensator is the nominal focusing system at pseudo-slit position. Regarding the aspheric, this lens is very close to the focal plane, thus very small footprint is on the lens and relaxed specifications are possible. No interferometric testing is required (the aspherical departure is too large) and mechanical perfilometry is enough for the considered error. Postpolishing will be applied in case $\mathrm{CNC}$ is used for the surface generation. A total of $200 \mathrm{MC}$ were run starting with a MF of $0.0846 \mathrm{~mm} \mathrm{rms}$. The $90 \%$ of the systems were below $0.0868 \mathrm{~mm} \mathrm{rms}$, being the degradation $0.00258 \mathrm{~mm} \mathrm{rms}$.

Regarding the camera, the as-built measured data will be reported in order to compute the optimum position of CAM S-5 and focal position. A total of $200 \mathrm{MC}$ were run starting with a MF of $0.00846 \mathrm{~mm} \mathrm{rms}$. The $90 \%$ of the systems were below $0.0887 \mathrm{~mm}$ rms, being the degradation $0.00295 \mathrm{~mm}$ rms. The lens CAM-S5 (camera compensator) would have a de-centering range $<0.25 \mathrm{~mm}$, a position range $< \pm 1.6 \mathrm{~mm}$ and a focal position range $< \pm 0.11 \mathrm{~mm}$.

Assembly errors: Once the singlets are manufactured, the lenses are glued in doublets. The tilts or decentering in the glued cavities of the singlets have not been considered as these have minimum effects on the performance with the used tolerances. Notice that positioning errors in the barrel (along axis) are considered in the unknown errors.

Regarding the Collimator, the tilt tolerance for all lenses within the barrel (in both $\mathrm{X}$ and $\mathrm{Y}$ ) is $< \pm 2.1$ arcmin and the decenter tolerance $< \pm 0.3 \mathrm{~mm}$. The worst case for total tilt is $3 \mathrm{arcmin}$. The collimators elements are not worst offenders in the system. A total of $200 \mathrm{MC}$ were run starting with a MF of $0.0846 \mathrm{~mm} \mathrm{rms}$. The $90 \%$ of the systems were below $0.0868 \mathrm{~mm} \mathrm{rms}$, being the degradation $0.00195 \mathrm{~mm} \mathrm{rms}$. The focus compensator range used is below $\pm 0.02 \mathrm{~mm}$.

Regarding the camera, the tilt tolerance for all lenses - except CAM-S5, within the barrel (X and Y) is $< \pm 2.1$ arcmin and the de-center tolerance $< \pm 0.15 \mathrm{~mm}$. The compensator for the camera is CAM-S5, whose position will be adjusted after manufacturing. The centering of CAM-S5 will be done with fine adjustment lateral screws with the camera under interferometric inspection to remove non-symmetrical aberrations on axis. In this case the total tilt of the elements is 3 arcmin (decomposed in $\mathrm{X}$ and $\mathrm{Y}$ tilts). A total of $200 \mathrm{MC}$ were run starting with a MF of $0.008460 \mathrm{~mm} \mathrm{rms}$. The $90 \%$ of the systems were below $0.0090 \mathrm{~mm} \mathrm{rms}$, being the degradation $0.0031 \mathrm{~mm} \mathrm{rms}$. De-centering range of CAM-S5 is below $0.31 \mathrm{~mm}$ in any axis (X-Y). The focusing range (at the pseudo-slit) used for camera adjustments is below $\pm 0.012 \mathrm{~mm}$. 
Uncompensated errors: These errors are referred to the unknown errors of the parameters that cannot be compensated except with the final instrument focus. ROC error is assumed to be $< \pm 0.02 \mathrm{~mm}$ and thickness $\pm<0.0 \mathrm{~mm}$. Compensator de-centering precision is assumed to be $< \pm 0.005 \mathrm{~mm}$ and lens position in barrel $< \pm 0.1 \mathrm{~mm}$. The compensator decentering tolerance is given by the positioning resolution that will be used to adjust CAM-S5. We are considering that this is the maximum centering precision that can be done. A total of $200 \mathrm{MC}$ were run starting with a MF of $0.00846 \mathrm{~mm}$ rms. The $90 \%$ of the systems were below $0.0092 \mathrm{~mm} \mathrm{rms}$, being the degradation $0.0036 \mathrm{~mm}$ rms. Collimator focusing range used in this simulation was $< \pm 0.5 \mathrm{~mm}$. The final errors will be provided by INAOE after manufacturing.

Thermal errors: We have evaluated the thermal effects regarding the image quality at the detector focal plane. The nominal model at $20^{\circ} \mathrm{C}$ at $\mathrm{P}=1 \mathrm{~atm}$ was moved at $\mathrm{T}=-4^{\circ} \mathrm{C}$ and $\mathrm{T}=+20^{\circ} \mathrm{C}$ with $\mathrm{P}=0.77 \mathrm{~atm}$. The optimized design is reported at $0.77 \mathrm{~atm}$, although $\mathrm{AIV}$ will be done at $20^{\circ} \mathrm{C}$ and $1 \mathrm{~atm}$. Instrument focus will be the only active compensator for atmospheric pressure changes. For this issue we used the thermal tool available in Zemax. The thermal changes were done in dimensions: thickness (aluminum was considered between lenses), ROC and diameters and index glasses using the $\mathrm{dn} / \mathrm{dt}$ reported values in the glass catalogue used by Zemax from Ohara and Hellma. After a camera athermalization opto-mechanical design, the RMS spot radius was evaluated and is within the available budget.

Glass homogeneity: As we are not diffraction limited high-order errors due to refraction index inhomogeneity of the glasses are expected. At most we probably might have some wedge effects as the major error with no consequences on the PSF. We have considered the homogeneity in the A20 $\left(20 \times 10^{-6}\right)$ group from Ohara and $\pm 10^{-6}$ for $\mathrm{CaF}_{2}$ from Hellma Materials. The homogeneity is modeled considering the use of a parabolic degradation of the refraction index. In this model we will consider that $50 \%$ of the aberration can be compensated with focus. The sub-pupils used inside the block will have a lower de-phase effect according to this parabolic model as they are squared with the aperture. Finally the homogeneity value WFE P-V is multiplied by a factor 0.4 to deliver a mean rms WFE value. This model fits well some large blanks for some materials, it is not clear the accuracy for these small blanks obtained from the previous ones, as the small cross sections will not allow to see this parabolic model. The contribution of the index homogeneity for each optical component for the OPD has been computed as: $\mathrm{OPD}=0.5 \times \mathrm{F}^{2} \times$ Central Thickness $\mathrm{x}$ IndexHomog; where $\mathrm{F}$ is the used fraction of the aperture. The expected rms wavefront error is $274 \mathrm{~nm}$. The nominal design averaged at $480 \mathrm{~nm}$ has a wavefront rms of 1.03 waves, which is $652 \mathrm{~nm}$. So the homogeneity error would account for a degradation from $652 \mathrm{~nm}$ to $\left(652^{2}+274^{2}\right)^{1 / 2}=707 \mathrm{~nm}$ rms. This is an increase of $8.4 \%$ over the nominal criteria.

We use for a rough estimation the same degradation regarding the nominal RMS spot radius from $0.00846 \mathrm{~mm}$ to $0.0092 \mathrm{~mm}$. We will use a value of $0.0036 \mathrm{~mm}$ for this contribution. The A20 group is the most relaxed Ohara grade while the $\mathrm{CaF}_{2}$ at $\pm 10^{-6}$ could require using mono-crystalline $\mathrm{CaF}_{2}$ for the required sizes, which increases the price a large amount. At the end we plan to order the mono-crystalline blanks.

Other effects: we have also studied in detail the following effects as part of the Error Budget: detector flatness, pseudoslit curvature, focusing step and anamorphic effect, in order to take into account all possible offenders to image quality.

To finish the optical design, we have carried out a complete ghost and stray light analysis in each configuration and an athermalized camera opto-mechanical design, with FEM and thermal analyses. Finally, we have produced the interferograms of both, collimator and camera, as templates to test the final measured values. We have a complete set of more than 80 drawings for the complete MEGARA Optics.

\section{CONCLUSIONS}

MEGARA optical design fulfills all the scientific requirements. The $\mathrm{F} / \mathrm{C}$ optics have been design to balance the spaxel size related to the sky sampling on sky and the losses due to the pupil oversize needed to allow a correct flux calibration. A field lens has been included to correct from the lack of telecentricity. A set of microlenses allows the conversion between the telescope plate scale and f/ number to the required value for the fibers. Finally, the pseudo-slit design is made of individual boxes along the focal plane curvature in order to guarantee performance at the entrance focal plane.

The spectrograph is fed by an $\mathrm{f} / 3$ beam coming from the fiber exit at the pseudo-slit. The design is composed by a collimator and a camera (both refractive) with a fixed angle between the two elements. This design allows the wide required spectral resolution range with the three kinds of pupil elements: the LR gratings $(R=5,500)$, the MR units $(\mathrm{R}=10,000)$ and the HR elements $(\mathrm{R}=17,000)$. The baseline design projects the spectral/spatial resolution element on 4 
pixels for a $100 \mu \mathrm{m}$ fiber core, which is the case for the LCB and MOS modes, covering the $4 \mathrm{k} \mathrm{x} 4 \mathrm{k}$ detector. The use of the SCB and/or the MOS with a decker, decreases the entrance slit width, increasing resolving power by, at least, $15 \%$.

Pupil elements are based on VPH gratings. These gratings will be sandwiched between two flat windows (in the LR units) or two prisms (in the case of the MR units). For HR elements, the baseline is the use of a sandwich of the VPH between two prisms each side, using our novel design of sliced pupil gratings although single prisms combined with a decker at the position of the pseudo-slit is also considered.

The transmission of the optical system is very high and can even be optimized with a specific coating program that is carrying out by INAOE and CIO centers in Mexico. Regarding the image quality, the error budget associated to the current optical design has room enough to assure that the high-level scientific requirements will be fulfilled. Evaluation has been done for all VPHs, along the whole wavelength range and along the $110 \mathrm{~mm}$ pseudo-slit. The Error Budget margin predicts that the high spectral resolution expected with the SCB and/or the MOS with a decker shall be achieved.

We have completed the ghost, stray light and thermal analyses, whose results have been used to feedback the optomechanical design. A complete set of drawings has been produced and the project is ready to order the blanks, task in the critical path of the project. INAOE and CIO have prepared the manufacturing plan, which includes polishing and coating. Should the funds arrive on time; MEGARA will be ready to observe at GTC by the end of 2015.

\section{REFERENCES}

[1] Gil de Paz, A., et al., "MEGARA: the future optical IFU and multi-object spectrograph for the 10.4m GTC telescope," Proc. SPIE 8446, 4Q1-4Q9 (2012). doi:10.1117/12.925739.

[2] Pérez-Calpena, A., Arrillaga X., Gil de Paz, A., Sánchez-Blanco. E., García-Vargas, M.L., Carrera, M.A., Gallego, J., Carrasco E., Sánchez, F.M., Vílchez, J.M. \& MEGARA Team, "MEGARA focal plane subsystems," Proc. SPIE 8446, 5E1-5E15 (2012).

[3] Carrasco, E., Sánchez-Blanco, E., García Vargas, M.L., Gil de Paz A., Páez, G., Gallego, J., Sánchez, F.M., Vílchez, J.M and MEGARA Team, "MEGARA Spectrograph Optics," Proc. SPIE 8446, 5B1-5B13 (2012).

[4] Carrasco, E., Sánchez-Blanco, E., García Vargas, M.L., Gil de Paz A., Gallego, J., Páez, G., Zamorano, J. and Castro, J., "Optical design for MEGARA: a multi-object spectrograph for the GTC," Proc. SPIE 8011, 2D12D10 (2011). doi: 10.1117/12.903436

[5] Sánchez-Blanco, E., García-Vargas, M.L., Maldonado, M., Gallego, J., Gil de Paz, A., Carrasco, E., Pérez, A., Martínez-Delgado, I. and Zamorano J., "Sliced-pupil grating: a novel concept for increasing spectral resolution," Proc. SPIE 8011, 1I-1 - 1I-10 (2011). doi: 10.1117/12.903186

[6] García-Vargas, M.L., Pérez, A., Gallego, J., Gil de Paz, A., Sánchez-Blanco, E., Martínez-Delgado, I., Maldonado M. and Zamorano J., "VIENTOS: a feasibility study of innovative pupil systems for the new generation of instruments in the large telescopes," Proc. SPIE 8446, 7T1-7T15 (2012). doi:10.1117/12.926062.

\section{Acknowledgements}

MEGARA is being funded by GRANTECAN, public entity responsible for the management and operation of GTC. The sliced-pupil grating design efforts are part of a collaborative project between the SME FRACTAL and the UCM. The first prototype received funds from the Madrid Regional Government (CAM) through grant 22/2009 for Aero-spatial Innovation. The development of this concept for large telescopes (VIENTOS project) has been co-funded by CDTI (Development and Industrial Transfer Center; Ministry of Economy and Competiveness, Spain) under the Industry for Science program IdC-20101106. The authors thank also the support received from the Consolider-Ingenio 2010 Program grant CSD2006-00070 "First Science with the GTC" and the Spanish National Plan for Astronomy \& Astrophysics grant AyA2009-10368. The LICA-UCM laboratory is funded by the Moncloa "Campus de Excelencia Internacional". 